\title{
Multinomial Lagrange-Bernstein Approximants
}

\author{
Luc Knockaert, Senior Member, IEEE
}

\begin{abstract}
The well-known Bernstein polynomials are frequently used in signal representation, finite impulse response filter realization, computer-aided geometric design, and B-spline techniques. In this letter, a refinement of the Bernstein approximation scheme for complex exponentials, by making use of a judicious Lagrange interpolation scheme, is proposed. Applied to a general function, this approach leads to a new polynomial approximant, termed a multinomial Lagrange-Bernstein approximant, that performs better than the usual Bernstein approximant.
\end{abstract}

Index Terms-Approximation of exponentials, Bernstein polynomials, Lagrange interpolation, multinomials, signal representation.

\section{INTRODUCTION}

B ERNSTEIN polynomials are frequently used in signal representation [1], [2], digital finite impulse response (FIR) filter realization such as the design of maximally flat filters [3], [4], computer-aided geometric design [5], and higher order polynomial elements and B-splines [6]. In this letter, we show that the simple binomial Bernstein approximation scheme of a general complex exponential function can be generalized in a straightforward manner to a multinomial Lagrange-interpolation-based approximation of this same exponential function. Applied to more general functions, this then leads to a new refined polynomial approximation, called multinomial Lagrange-Bernstein approximation. Pertinent examples show that the new approximation technique performs better than the usual Bernstein approximation.

\section{APPROXIMATION OF EXPONENTIALS}

It is undergraduate knowledge that the exponential $e^{\alpha t}$, with $\alpha$ complex, can be defined as $\lim _{N \rightarrow \infty}(1+\alpha t / N)^{N}$. However, this does not provide a suitable approximation of any practical importance. A better approximation can be found as follows: since $\alpha / N \approx e^{\alpha / N}-1$, we can write

$$
e^{\alpha t} \approx\left(1-t+t e^{\frac{\alpha}{N}}\right)^{N}=\sum_{k=0}^{N} e^{\frac{k \alpha}{N}}\left(\begin{array}{l}
N \\
k
\end{array}\right) t^{k}(1-t)^{N-k}
$$

which is the well-known Bernstein polynomial approximation, uniformly convergent in $[0,1]$ as $N$ tends to infinity. Now the

Manuscript received September 22, 2005; revised January 6, 2006. The associate editor coordinating the review of this manuscript and approving it for publication was Dr. Israel Cohen.

The author is with the INTEC-IMEC-Ghent University, B-9000 Gent, Belgium (e-mail: luc.knockaert@intec.ugent.be).

Digital Object Identifier 10.1109/LSP.2006.871719 approximation (1) is in fact the $N$ th power of the linear interpolation formula with nodes 0 and 1 for $e^{\alpha t / N}$, i.e.,

$$
e^{\frac{\alpha t}{N}} \approx 1-t+t e^{\frac{\alpha}{N}}
$$

Therefore, we have the interesting fact that the crude linear interpolation formula (2), when raised to the $N$ th power, generates the nice uniform formula (1). As a generalization of the linear interpolant (2), we may take $M+1$ nodes $(M \geq 1)$

$$
0 \leq t_{0}<t_{1}<\cdots<t_{M} \leq 1
$$

and form the Lagrange interpolant for $e^{\alpha t / N}$ with respect to these nodes, i.e.,

$$
e^{\frac{\alpha t}{N}} \approx \sum_{k=0}^{M} e^{\frac{\alpha t_{k}}{N}} \ell_{k}(t)
$$

where

$$
\ell_{k}(t)=\frac{\prod_{l \neq k}\left(t-t_{l}\right)}{\prod_{l \neq k}\left(t_{k}-t_{l}\right)}
$$

and after raising to the $N$ th power, find the hopefully better formula

$$
e^{\alpha t} \approx\left(\sum_{k=0}^{M} e^{\frac{\alpha t_{k}}{N}} \ell_{k}(t)\right)^{N}
$$

Fixing the nodes $t_{k}$, it is therefore crucial to find estimates for the bounds of the error function

$$
R_{N}(t, \alpha, M)=e^{\alpha t}-\left(\sum_{k=0}^{M} e^{\frac{\alpha t_{k}}{N}} \ell_{k}(t)\right)^{N}
$$

over the interval $[0,1]$. We need the following.

Lemma: Let $m, \lambda \geq 0$. Then the function

$$
\kappa(\lambda, m)=\max _{x \geq 1} x^{m}\left(\left(1+\frac{\lambda}{x^{m+1}}\right)^{x}-1\right)
$$

is bounded such that $\lambda \leq \kappa(\lambda, m) \leq e^{\lambda}-1$.

Proof: It is easily seen that $\kappa(\lambda, 0)=e^{\lambda}-1$. For $m>0$, we have

$$
\begin{aligned}
& \lim _{x \rightarrow 1} x^{m}\left(\left(1+\frac{\lambda}{x^{m+1}}\right)^{x}-1\right) \\
& \quad=\lambda=\lim _{x \rightarrow \infty} x^{m}\left(\left(1+\frac{\lambda}{x^{m+1}}\right)^{x}-1\right) .
\end{aligned}
$$


The right-hand side of (9) follows from the fact that

$$
x \ln \left(1+\frac{\lambda}{x^{m+1}}\right) \approx \frac{\lambda}{x^{m}} \text { for } x \rightarrow \infty .
$$

Also, since

$$
\left(1+\frac{\lambda}{x^{m+1}}\right)^{x}-1 \leq e^{\frac{\lambda}{x^{m}}}-1
$$

we have that

$$
\kappa(\lambda, m) \leq \max _{x \geq 1} x^{m}\left(e^{\frac{\lambda}{x^{m}}}-1\right)=\max _{0 \leq u \leq 1} \frac{e^{\lambda u}-1}{u}=e^{\lambda}-1
$$

which completes the proof.

The lemma enables us to state the following.

Theorem 1: There is a constant $K(\alpha, M)$ such that

$$
\max _{0 \leq t \leq 1}\left|R_{N}(t, \alpha, M)\right| \leq K(\alpha, M) N^{-M} .
$$

Proof: Recall that the error function $R_{N}(t, \alpha, M)$ is given by

$$
R_{N}(t, \alpha, M)=e^{\alpha t}-\left(\sum_{k=0}^{M} e^{\frac{\alpha t_{k}}{N}} \ell_{k}(t)\right)^{N}
$$

From

$$
R_{1}(t, \alpha, M)=e^{\alpha t}-\sum_{k=0}^{M} e^{\alpha t_{k}} \ell_{k}(t)
$$

we deduce that

$$
R_{N}(t, \alpha, M)=e^{\alpha t}-\left(e^{\frac{\alpha t}{N}}-R_{1}\left(t, \frac{\alpha}{N}, M\right)\right)^{N}
$$

and hence

$\left|R_{N}(t, \alpha, M)\right| \leq\left|e^{\alpha t}\right| \times\left[\left(1+\left|e^{\frac{-\alpha t}{N}} R_{1}\left(t, \frac{\alpha}{N}, M\right)\right|\right)^{N}-1\right]$.

Now by virtue of Kowalewski's exact remainder theorem [7, p. 71], we have

$$
e^{-\alpha t} R_{1}(t, \alpha, M)=\frac{\alpha^{M+1}}{M !} \sum_{k=0}^{M} \ell_{k}(t) \int_{t_{k}}^{t}\left(t_{k}-\tau\right)^{M} e^{\alpha(\tau-t)} d \tau .
$$

Introducing the Lebesgue constant [8]

$$
\Lambda_{M}=\max _{0 \leq t \leq 1} \sum_{k=0}^{M}\left|\ell_{k}(t)\right|
$$

we obtain the bound

$$
\begin{aligned}
\left|e^{-\alpha t} R_{1}(t, \alpha, M)\right| \leq & \frac{|\alpha|^{M+1}}{M !} \times \Lambda_{M} \\
& \times \max _{0 \leq u, t \leq 1}\left|\int_{u}^{t}(u-\tau)^{M} e^{\alpha(\tau-t)} d \tau\right|
\end{aligned}
$$

and since

$$
\max _{0 \leq u, t \leq 1} \int_{u}^{t}|u-\tau|^{M} d \tau \times \max _{0 \leq t, \tau \leq 1}\left|e^{\alpha(\tau-t)}\right| \leq \frac{e^{|\Re \alpha|}}{M+1}
$$

where $\Re \alpha$ is the real part of $\alpha$, we find

$$
\left|e^{-\alpha t} R_{1}(t, \alpha, M)\right| \leq \frac{|\alpha|^{M+1}}{(M+1) !} \Lambda_{M} e^{|\Re \alpha|} .
$$

Inserting this in (17), we obtain

$$
\begin{aligned}
\left|R_{N}(t, \alpha, M)\right| \leq\left|e^{\alpha t}\right| & \\
\times & {\left[\left(1+\frac{|\alpha|^{M+1}}{N^{M+1}} \frac{\Lambda_{M}}{(M+1) !} e^{\frac{|\Re \alpha|}{N}}\right)^{N}-1\right] . }
\end{aligned}
$$

Finally, putting

$$
\lambda(\alpha, M)=\frac{|\alpha|^{M+1} \Lambda_{M} e^{|\Re \alpha|}}{(M+1) !}
$$

and considering that $\left|e^{\alpha t}\right| \leq e^{\max (0, \Re \alpha)}$, we obtain, by virtue of the lemma, that the theorem is satisfied if we take

$$
K(\alpha, M)=e^{\max (0, \Re \alpha)} \kappa(\lambda(\alpha, M), M) .
$$

This completes the proof.

Note that the presence of the Lebesgue constant $\Lambda_{M}$ indicates that we should not take the equispaced nodes $t_{k}=k / M$, yielding a rather large $\Lambda_{M}$, but instead some kind of Chebyshev nodes, such as the extended Chebyshev nodes

$$
t_{k}=\frac{1}{2}-\frac{\cos \left[\frac{(2 k+1) \pi}{(2 M+2)}\right]}{2 \cos \left[\frac{\pi}{(2 M+2)}\right]} \quad k=0, \ldots, M
$$

which yield a much more moderate Lebesgue constant for large $M$ [8]. For the simplest cases $M=1$ and $M=2$, this yields, respectively, the nodes $(0,1)$ and $(0,1 / 2,1)$, which, for these low values of $M$, are nevertheless exceptionally equispaced.

\section{Multinomial Lagrange-Bernstein ApPROXimants}

Since Theorem 1 shows that the error function $R_{N}(t, \alpha, M)$ tends to zero as $N$ tends to $\infty$, this implies that the polynomial of the $N \times M$ th degree

$$
E_{N}(t, \alpha, M)=\left(\sum_{k=0}^{M} e^{\frac{\alpha t_{k}}{N}} \ell_{k}(t)\right)^{N}
$$


is a good approximation in [0,1] for the exponential $e^{\alpha t}$. The polynomial $E_{N}(t, \alpha, M)$ can be expanded multinomially as

$$
\begin{aligned}
E_{N}(t, \alpha, M)= & \sum_{\sum_{k=0}^{M} m_{k}=N}\left(\begin{array}{c}
N \\
m_{0}, m_{1}, \ldots, m_{M}
\end{array}\right) \\
& \times e^{\frac{\alpha\left(\sum_{k=0}^{M} m_{k} t_{k}\right)}{N}} \times \prod_{k=0}^{M} \ell_{k}(t)^{m_{k}}
\end{aligned}
$$

where the multinomial coefficient is defined as

$$
\left(\begin{array}{c}
N \\
m_{0}, m_{1}, \ldots, m_{M}
\end{array}\right)=\frac{N !}{\prod_{k=0}^{M} m_{k} !} .
$$

In is seen in (28) that the exponential $e^{\alpha t}$ is sampled at the points $\left(\sum_{k=0}^{M} m_{k} t_{k}\right) / N$. Applied to a more general function $f(t)$, we can therefore define the multinomial Lagrange-Bernstein approximant $\tilde{f}_{N, M}(t)$ of $f(t)$, namely, the polynomial of the $N \times M$ th degree

$$
\begin{aligned}
\tilde{f}_{N, M}(t)=\sum_{\sum_{k=0}^{M} m_{k}=N}\left(\begin{array}{c}
N \\
m_{0}, m_{1}, \ldots, m_{M}
\end{array}\right) \\
\times f\left(\frac{1}{N} \sum_{k=0}^{M} m_{k} t_{k}\right) \times \prod_{k=0}^{M} \ell_{k}(t)^{m_{k}} .
\end{aligned}
$$

Note that for $M=1, t_{0}=0$, and $t_{1}=1$, we have the simple Bernstein polynomial approximant. Regarding the general multinomial Lagrange-Bernstein approximant $\tilde{f}_{N, M}(t)$, we can state the following two theorems.

Theorem 2: Let $f(t)$ be a polynomial of degree at most $M$. Then $\tilde{f}_{N, M}(t)=f(t)$.

Proof: From $R_{1}(t, \alpha, M)=\mathcal{O}\left(\alpha^{M+1}\right)$ for $\alpha$ tending to zero, independently of $t$, it follows that

$$
\begin{aligned}
R_{N}(t, \alpha, M) & =e^{\alpha t} \times\left[1-\left(1-e^{-\frac{\alpha t}{N}} R_{1}\left(t, \frac{\alpha}{N}, M\right)\right)^{N}\right] \\
& =\mathcal{O}\left(\alpha^{M+1}\right) .
\end{aligned}
$$

Hence the 0th, first, $\ldots, M$ th derivatives of $R_{N}(t, \alpha, M)=$ $e^{\alpha t}-E_{N}(t, \alpha, M)$ with respect to $\alpha$ at $\alpha=0$ must vanish. This completes the proof.

Theorem 3: Suppose that $f(t)$ can be written as

$$
f(t)=\sum_{k=1}^{\infty} c_{k} e^{\alpha_{k} t} \quad 0 \leq t \leq 1
$$

Then $\tilde{f}_{N, M}(t) \rightarrow f(t)$, uniformly in [0,1], as $N$ tends to infinity, provided

$$
\sum_{k=1}^{\infty}\left|c_{k}\right| K\left(\alpha_{k}, M\right)<\infty
$$

where $K(\alpha, M)$ is as defined in Theorem 1 .

Proof: It is a simple matter to show that

$$
f(t)-\tilde{f}_{N, M}(t)=\sum_{k=1}^{\infty} c_{k} R_{N}\left(t, \alpha_{k}, M\right)
$$

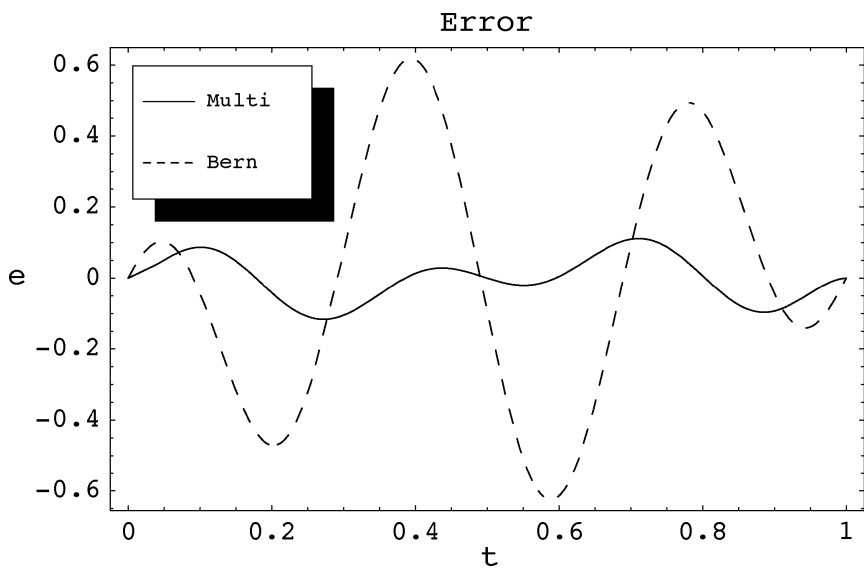

Fig. 1. Multinomial error versus the Bernstein error for the first example.

and hence

$$
\left|f(t)-\tilde{f}_{N, M}(t)\right| \leq N^{-M} \sum_{k=1}^{\infty}\left|c_{k}\right| K\left(\alpha_{k}, M\right)
$$

which proves the theorem.

Important Remark: It is seen that the convergence is $\mathcal{O}\left(N^{-M}\right)$, provided that requirement (33) is satisfied. This is certainly the case when the number of exponentials is finite (see, e.g., the first example below).

In general, the function $f(t)$ must be expandable as an infinite sum of exponentials. This presupposes that this set of exponentials is complete in some Banach space defined over the interval $[0,1]$. This is the case for large classes of exponentials: see, e.g., Raymond Redheffer's extended paper [9] and Robert Young's well-known book [10]. Also, it is difficult in the general case to test requirement (33), due to the complicated form of the function $K(\alpha, M)$. Nevertheless, as can be seen from the second example below, the multinomial Lagrange-Bernstein approximant performs quite well, even in the general case.

\section{A. Examples}

Here we will compare the Bernstein approximant $\tilde{f}_{32,1}(t)$ (with nodes $t_{0}=0$ and $t_{1}=1$ ) with the multinomial Lagrange-Bernstein approximant $\tilde{f}_{16,2}(t)$ (with nodes $t_{0}=0$, $t_{1}=1 / 2$, and $t_{2}=1$ ) for two pertinent functions. Note that both $\tilde{f}_{32,1}(t)$ and $\tilde{f}_{16,2}(t)$ are polynomials of degree 32 .

As a first example, we take $f(t)=\cos (16 t)=(1 / 2) e^{16 i t}+$ $(1 / 2) e^{-16 i t}$. Fig. 1 shows the multinomial Lagrange-Bernstein error $f(t)-\tilde{f}_{16,2}(t)$ versus the Bernstein error $f(t)-\tilde{f}_{32,1}(t)$.

As a second example, we take the piecewise linear function defined as

$$
\begin{array}{ll}
f(t)=0 & t \in[0,0.25] \\
f(t)=4 t-1 & t \in[0.25,0.5] \\
f(t)=5-8 t & t \in[0.5,0.75] \\
f(t)=4 t-4 & t \in[0.75,1] .
\end{array}
$$

Note that this function is not expandable as a finite sum of exponentials. Figs. 2 and 3, respectively, show the Bernstein approx- 


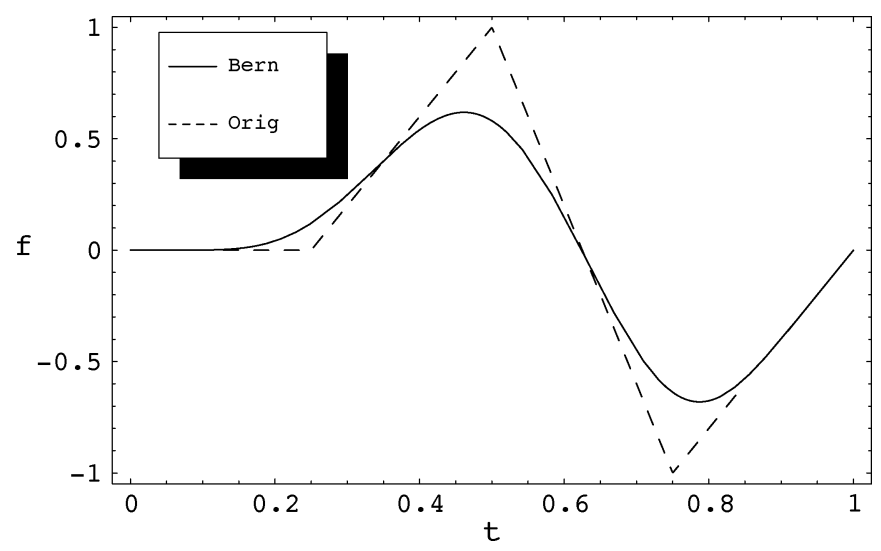

Fig. 2. Bernstein approximant versus the function for the second example.

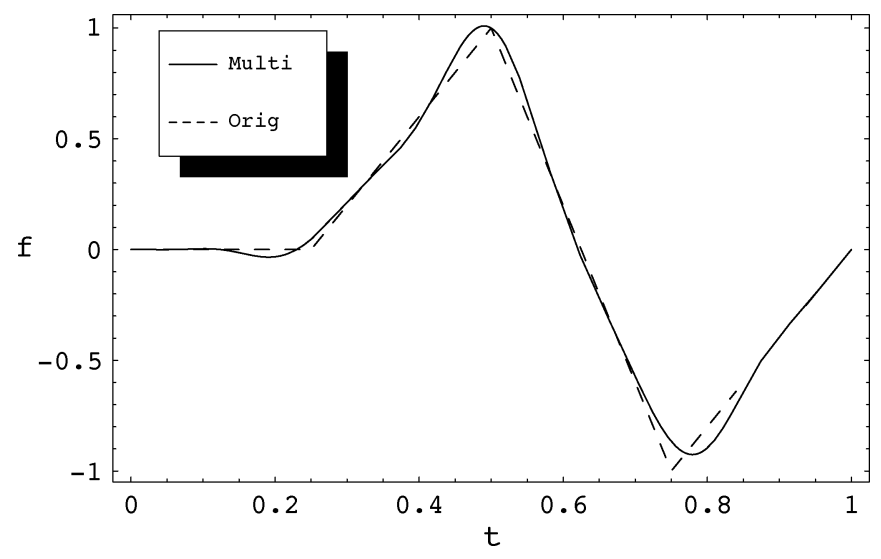

Fig. 3. Multinomial approximant versus the function for the second example.

imant $\tilde{f}_{32,1}(t)$ versus $f(t)$ and the multinomial Lagrange-Bernstein approximant $\tilde{f}_{16,2}(t)$ versus $f(t)$.

It is seen in both examples that the multinomial Lagrange-Bernstein approximant is significantly closer to the original function than the simple Bernstein approximant.

\section{CONCLUSION AND FUture RESEARCH}

We showed that the Bernstein polynomial approximation of a complex exponential can be generalized to what we coined the multinomial Lagrange-Bernstein approximation of this same exponential. Under certain conditions, this leads to a new refined polynomial approximation for more general functions, which performs better than the simple Bernstein approximation.

Since the Bernstein approximation is useful for its smooth approximation properties, and in particular gives smooth frequency response functions in filter design, the same can be expected of the multinomial Lagrange-Bernstein technique, especially since some of the underlying Lagrange nodes can be freely chosen.

However, it is well known that the Bernstein approximation is not very good in terms of $L_{2}$ or Chebyshev approximation. A possible solution would be to abandon the Bernstein approach (which is interpolatory) altogether for the related Durrmeyer approach [11], which is much more $L_{2}$ projection related, and look for appropriate refinements of the Durrmeyer technique. In the same vein, it would be tempting to apply similar refining approaches to other polynomial or spline bases. This is the purpose of ongoing research.

\section{ACKNOWLEDGMENT}

The author would like to thank the anonymous reviewers for their suggestions, which allowed him to greatly enhance the quality of the manuscript.

\section{REFERENCES}

[1] S. Samadi, "Explicit formula for improved filter sharpening polynomial," IEEE Trans. Signal Process., vol. 9, no. 10, pp. 2957-2959, Oct. 2000.

[2] D. B. H. Tay, "Zero-pinning the Bernstein polynomial: A simple design technique for orthonormal wavelets," IEEE Signal Process. Lett., vol. 12 , no. 12 , pp. 835-838, Dec. 2005.

[3] L. R. Rajagopal and S. C. Dutta Roy, "Design, of maximally-flat FIR filters using the Bernstein polynomial," IEEE Trans. Circuits Syst., vol. CAS-34, no. 12, pp. 1587-1590, Dec. 1987.

[4] T. Cooklev, T. Yoshida, and A. Nishihara, "Maximally flat half-band diamond-shaped FIR filters using the Bernstein polynomial," IEEE Trans. Circuits Syst. II, vol. 40, no. 11, pp. 749-751, Nov. 1993.

[5] G. Farin, Curves and Surfaces for Computer Aided Geometric Design. A Practical Guide. Boston, MA: Academic, 1988.

[6] S. Samadi, O. Ahmad, and M. N. S. Swamy, "Characterization of B-spline digital filters," IEEE Trans. Circuits Syst. I, Reg. Papers, vol. 51, no. 4, pp. 808-816, Apr. 2004.

[7] P. J. Davis, Interpolation \& Approximation. New York: Dover, 1975.

[8] L. Brutman, "Lebesgue functions for polynomial interpolation-a survey," Ann. Numer. Math., no. 4, pp. 111-127, 1997.

[9] R. M. Redheffer, "Completeness of sets of complex exponentials," $A d v$. Math., vol. 24, no. 1, pp. 1-62, 1977.

[10] R. M. Young, An Introduction to Nonharmonic Fourier Series, revised 1st ed. San Diego, CA: Academic, 2001.

[11] J. C. Sevy, "Lagrange and least-squares polynomials as limits of linear combinations of iterates of Bernstein and Durrmeyer polynomials," $J$. Approx. Theory, no. 80, pp. 267-271, 1995. 